\title{
A patient with Rotor syndrome and coronary artery disease: is it a coincidental or related?
}

\author{
Lila Kumaladewi ${ }^{\mathrm{a}}$, Herry Purbayu ${ }^{\mathrm{b}}$ \\ ${ }^{\mathrm{b}}$ Email: herrypsby@gmail.com

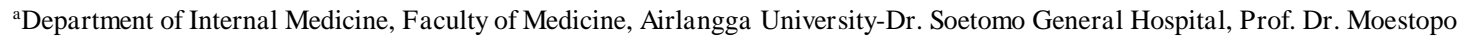 \\ St. No 6-8, Surabaya 60286, Indonesia \\ ${ }^{\mathrm{a}}$ Email: lilakumala@gmail.com \\ ${ }^{b}$ Division of Gastroentero-hepatology, Department of Internal Medicine, Faculty of Medicine, Airlangga University-Dr. Soetomo \\ General Hospita1, Prof. Dr. Moestopo St. No 6-8, Surabaya 60286, Indonesia
}

\begin{abstract}
Background: Rotor syndrome is a rare, benign and inherited cause of chronic hyperbilirubinemia. Several studies have provided evidence that elevated bilirubin level has cardioprotective benefits with reduction in the severity of coronary artery disease (CAD). However, current evidence is inconclusive regarding the protective effect of bilirubin on CAD. Case description: A 57-year-old male with a history of chronic jaundice since childhood, presents to the Gastroenterohepatology clinic of Dr. Soetomo General Hospital with CAD Triple Vessel Disease pro urgent CABG. Physical examination revealed icteric sclerae without hepatosplenomegaly. The patient suffered from hypertension and dyslipidemia for 10 years. He had an elevated total bilirubin level of $5.92 \mathrm{mg} / \mathrm{dL}$ with a direct bilirubin of $4.02 \mathrm{mg} / \mathrm{dL}$ ( $68 \%$ of total bilirubin), with normal aspartate aminotransferase (AST), alanine aminotransferase (ALT) and alkaline phosphatase (ALP) levels. An evaluation for chronic liver disease revealed negative hepatitis serologies, anti-smooth muscle antibody, antinuclear antibody and antimitochondrial antibody as well as normal. BSP clearance test results show a delay. Abdomen computed tomography (CT) scan showed normal without any biliary dilation. The liver biopsy was normal with no hepatocellular pigment.

Conclusion: A patient with chronic jaundice symptoms has been reported who is later diagnosed as Rotor syndrome. The patient also suffered from CAD. The protective effects of bilirubin are not proven in this patient.
\end{abstract}

Keywords: Rotor syndrome; bilirubin; antioxidant activity; coronary artery disease; cardiovascular disease.

\section{Introduction}

Rotor syndrome is the second most rare hereditary disease cause of hyperbilirubinemia ((Erlinger et al., 2014); (Pratt et al., 2012)). Typically, patients are asymptomatic, and the predominantly conjugated hyperbilirubinemia are often discovered incidentally. This phenomenon is a result of impaired hepatocellular storage of conjugated bilirubin that leaks into plasma causing hyperbilirubinemia. Its presenting symptom is jaundice, but Rotor Syndrome is a benign, self-limiting disorder that does not require treatment (van de Steeg et al., 2012).

Elevated serum bilirubin has been associated with reduced risk of CAD. The available evidence regarding the effects of bilirubin are not definitive and different studies provide contradictory findings (Gupta et al., 2017). Whether bilirubin is a byproduct of another reaction with cardioprotective effects, or whether it has a direct role in reducing severity of CAD remains to be elucidated (Acet et al., 2014). 
A 57-year-old male patient was consulted from the cardiology department to the Gastroenterohepatology outpatient clinic of Dr. Soetomo General Hospital with icterus pro evaluation and coronary artery disease triple vessel disease (CAD TVD) pro urgent coronary artery bypass graft (CABG). He has a history of chronic jaundice since early childhood. He complained yellowish skin and eyes since the age of 1 year. He also complained dark color urine. No complain of nausea, vomiting, itchy, abdominal pain, fever, general weakness and pale stool. No history of coffee grounds-like and bloody vomiting. He was not on any hepatotoxic medication. He suffered from hypertension and dyslipidemia for 10 years. No history of hepatitis and autoimmune disease. No history of his family member experiences chronic jaundice like him. The patient had heart attack, diagnosed as CAD TVD on September 29th, 2017 and underwent percutaneous transluminal coronary angioplasty (PTCA) stent. Staging percutaneous coronary intervention (PCI) was performed on December 29th, 2017 showed coronary artery restenosis. The patient visit cardiology clinic routinely, last medication was ASA100mg q.d., clopidogrel 75mg q.d., lisinopril10mg q.d., ISDN $5 \mathrm{mg}$ b.i.d., bisoprolol $2.5 \mathrm{mg}$ b.i.d. and atorvastatin $40 \mathrm{mg}$ q.d.

Patient had compos mentis consciousness, visual analog scale (VAS) of 0. Blood pressure was $136 / 84 \mathrm{mmHg}$, heart rate 70 beat/minute, respiratory rate 18 times/minute, temperature $36.6^{\circ} \mathrm{C}$ and $\mathrm{O} 2$ saturation $99 \%$. The patient had icteric sclera. No spider naevi, hepatosplenomegaly, ascites, collateral vein, erythema palmaris, and edema.

Automated blood count on April 17th, 2017, demonstrated hemoglobin of $15.5 \mathrm{~g} / \mathrm{dl}$, WBC

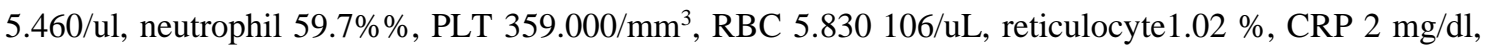
LED 29 mm/h, RBG 98 mg/dl, BUN 9 mg/dl, SC 0.80 mg/dl, AST 16 u/l, ALT 15 u/l, total bilirubin 5.92 $\mathrm{mg} / \mathrm{dl}$, direct bilirubin $4.02 \mathrm{mg} / \mathrm{dl}$, albumin serum 4,5 g/dl, ALP 49 u/L, PPT 10.5(11,3) seconds, APPT 27,1(25,7) seconds and AFP 5ng/ml. Protein electrophoresis within normal limits. Total Cholesterol was $182 \mathrm{mg} / \mathrm{dl}$, triglyceride $161 \mathrm{mg} / \mathrm{dl}$, HDL $35 \mathrm{mg} / \mathrm{dl}$ and LDL 130mg/dl. Serological examination of hepatitis showed HBsAg, anti-HBC and IgM anti-HAV non-reactive. ANA test, anti-smooth muscle antibody, and antimitochondrial antibody as well as normal. Urinalysis showed dark color, bilirubin +1 , Urobilin $1 \mathrm{mg} / \mathrm{dl}$, no blood, leukocyte, erythrocyte, leukocyte, cast, protein, glucose and ketone. BSP Tolerance test showed $52.63 \%$ (30 minute), $43.21 \%$ (45 minute), 26.31 (90 minute), 27.66 (120 minute).

Chest X Ray showed cor and lung within normal limit. Electrocardiography showed sinus rhytme $70 \mathrm{x} / \mathrm{minute}$, frontal and horizontal axis were normal. Abdominal ultrasound showed normal size, sharp angles, flat edge, the intensity of echo parenchyma appears to be normal homogeneous, not visible widening of the IHBD and EHBD, portal vein and hepatic vein looks normal, no nodules, cysts and masses appear. Normal size gallbladder, no wall thickness, no stone, nodules, sludge and mass. No extraluminal free fluid echo intensity was seen in the abdominal cavity. The liver, spleen, pancreas, kidney do not appear abnormalities. Abdominal MSCT Abdomen with and without contrast showed normal size liver, sharp angles, flat edge, normal density, not visible widening of the IHBD and EHBD, portal vein and hepatic vein looks normal, no nodules, cysts and masses appear. Gallbladder has normal size, normal density, no stone, nodules, sludge and mass. Liver biopsy showed normal parenchyma cell, no inflammation, fibrosis and hepatocellular pigment. Typical signs of intracellular virus infection were not found. 
Angiography on September 29th, 2017 showed stenosis 30\% in distal left main coronary artery (LMCA), diffuse disease heavy calcification in osteal-distal left anterior descending artery (LAD), stenosis $75 \%$ in proximal LAD, chronic total occlusion (CTO) in mid LAD, stenosis $90 \%$ in distal left circumflex artery (LCx) and no canalization in right coronary artery (RCA). It was concluded as CAD TVD, CTO in mid LAD and post PTCA stent in osteal-mid LAD. Angiography (staging PCI) on December 29th, 2017 showed stenosis 50-70\% in distal LMCA, in stent restenosis (ISR) osteal-mid LAD, CTO in mid LAD, significant stenosis 80-90\% in distal LCx and CTO in mid RCA. The second angiography was concluded as CAD TVD, CTO in mid RCA, ISR 90-99\% in osteal-mid LAD and CTO in mid LAD.

Patients diagnosed with Rotor syndrome, CAD TVD, left main disease, and it was decided to perform $\mathrm{CABG}$. This patient was not given therapy for Rotor syndrome. The medications from the cardiology clinic was continued. Patients and family members were reassured that Rotor syndrome is benign with no effect on life expectancy.

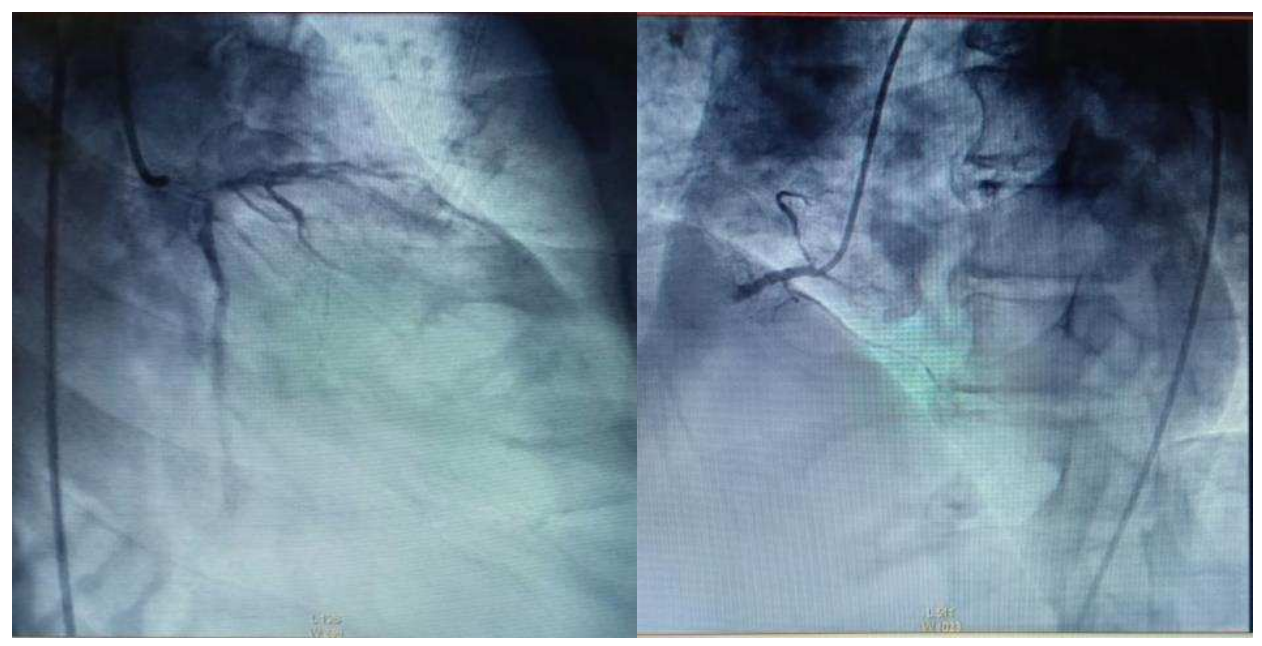

Figure 1. (Left)DCA (PCI staging on 09-29-2017); (Right) DCA (PCI staging on 29 -12- 2017)

\section{Research Methods}

\section{Research Results and Discussion}

Rotor syndrome is an autosomal recessive disorder caused by homozygous mutations in both the SLCO1B1 and SLCO1B3 genes on chromosome 12. These genes provide instructions for making organic anion transporting polypeptide 1B1 and 1B3 (OATP1B1 and OATP1B3, respectively). These proteins are found in liver cells and mediate sodium-independent cellular uptake of compounds including bilirubin glucuronide, bile acids, and steroid and thyroid hormones as well as numerous drugs, toxins, and their conjugates. In a normal liver, a majority of bilirubin is conjugated by hepatocytes and secreted back into the blood. It is then reabsorbed in downstream hepatocytes by the OATP1B1 and OATP1B3 proteins. In Rotor syndrome, the OATP1B1 and OATP1B3 proteins are abnormally short, therefore, the bilirubin is less efficiently taken up by the liver and removed from the body, causing a build-up of bilirubin in the blood and urine which results in jaundice and dark urine ((Erlinger et al., 2014); (Kagawa et al., 2017); (Pratt et al., 2012); (van de Steeg et al., 2012)). 
As mentioned, Rotor syndrome is caused by mutations in two proteins responsible for transporting bilirubin and other compounds from the blood to the liver to be metabolized and cleared from the body. Coproporphyrin I, a major coproporphyrin isomer in bile, is transported from the hepatocyte back into the circulation and is excreted in the urine. Thus, urine coproporphyrin is elevated in Rotor syndrome. Cholescintigraphy using sulfobromophthalein (BSP) have shown that the transport capacity of dye into bile is reduced by less than $50 \%$, and the storage capacity in the hepatocytes is decreased more than 5 -fold compared with normal values in this disease (Kagawa et al., 2017).

Patients with Rotor syndrome are typically asymptomatic, however, generalized non-pruritic jaundice can present at birth or early childhood. These symptoms may come and go. Some patients only have scleral icterus. Patients will also complain about passing dark-colored urine. Additionally, around 5\% to $30 \%$ of patients may also experience abdominal pain, gastric mucosal abnormalities, and fever (Memon et al., 2016).

Rotor syndrome is largely a diagnosis of exclusion. Serological abnormalities in Rotor syndrome only include elevated total serum bilirubin (typically elevated between 2 to $5 \mathrm{mg} / \mathrm{dL}$ but may be as high as $20 \mathrm{mg} / \mathrm{dL}$ ). Most of the time, alanine aminotransferase, aspartate aminotransferase, gamma-glutamyl transferase, and alkaline phosphatase levels are normal, but mild elevations can be seen. If any of these lab values are markedly elevated, investigation for other, more serious conditions is warranted. Imaging studies cannot diagnose Rotor syndrome but can help rule out other diseases that cause hyperbilirubinemia. For example, ultrasound of the liver and biliary tree can help investigate the causes of extra-hepatic biliary obstruction. The gallbladder is visualized on oral cholecystography in Rotor syndrome while it is not visualized in Dubin-Johnson syndrome (DJS). Ultimately, the best method of diagnosing the disease is the analysis of urine coproporphyrin excretion. The total urine coproporphyrin excretion in Rotor syndrome has a 2 to 5-fold elevation, with 65\% constituting coproporphyrin I (Memon et al., 2016).

Liver biopsy is not required to make the diagnosis of Rotor syndrome, but if done, liver biopsy in patients with the disease reveals normal histology. Liver biopsy may be helpful in distinguishing Rotor syndrome for other, more serious liver diseases. Since Rotor syndrome is clinically similar to DJS, it is imperative to distinguish between these two conditions. The absence of dark melanin-like pigments on liver biopsy distinguishes Rotor Syndrome from DJS (Dhumeaux and Erlinger 2013; Jirsa et al., 2012; Memon et al., 2016). This patient had icteric sclerae without hepatosplenomegaly. He suffered from hypertension and dyslipidemia for 10 years. His father also experiences chronic jaundice. He had an elevated total bilirubin level of $5.92 \mathrm{mg} / \mathrm{dL}$ with a direct bilirubin of $4.02 \mathrm{mg} / \mathrm{dL}$ ( $68 \%$ of total bilirubin), with normal AST, ALT and ALP levels. An evaluation for chronic liver disease revealed negative hepatitis serologies, anti-smooth muscle antibody, antinuclear antibody and antimitochondrial antibody as well as normal. BSP clearance test results show a delay. CT scan was normal without any biliary dilation. The liver biopsy was normal with no hepatocellular pigment.

Rotor syndrome is a benign disease requiring no treatment. Jaundice is a lifelong finding, but the disease is not associated with morbidity or mortality, and life expectancy is not affected. Most individuals with Rotor syndrome are born to consanguineous couples, and its diagnosis may coincidently identify consanguinity. Distinguishing Rotor syndrome from other more serious disorders is important to avoid 
unnecessary workup and interventions. It is also critical to reassure and calm patients or family members of patients with Rotors syndrome that the condition is benign ((Dhumeaux \& Erlinger, 2013); (Kagawa et al., 2017); (Shimizu et al., 1981)).This patient was not given therapy for Rotor syndrome. Reassure patient and family members that Rotor syndrome is benign with no effect on life expectancy and the occasional appearance of jaundice is important.

The many causes of hyperbilirubinemia can be divided into causes of unconjugated versus conjugated hyperbilirubinemia and then further classified. Some conditions that can cause hyperbilirubinemia include Dubin-Johnson syndrome, Gilbert syndrome, Crigler-Najjar syndrome (type I and type II), extra-hepatic biliary obstruction, familial intra-hepatic cholestasis, benign recurrent intrahepatic cholestasis (BRIC), drug-induced hepatotoxicity, hemolysis, cholestasis of pregnancy, viral hepatitis, autoimmune hepatitis, Wilson disease, hemochromatosis, alpha-1-antitrypsin deficiency and cirrhosis. It is important to differentiate Rotor syndrome from other diseases causing hyperbilirubinemia. Normal levels of alkaline phosphatase and gamma-glutamyltranspeptidase help distinguish Rotor syndrome from disorders associated with biliary obstruction. Abnormal urinary coproporphyrin excretion and normal liver histology help distinguish this entity from DJS ((van de Steeg et al., 2012); (Shimizu et al., 1981)).

Rotor syndrome is a benign disease with no effect on life expectancy. No adverse drug effects have been documented in people with Rotor syndrome, but the absence of the hepatic proteins OATP1B1 and OATP1B3 may lead to serious issues with liver uptake. OATP1B1 plays a role in drug detoxification, and with a reduced activity of this protein, certain drugs such as anticancer agents, methotrexate, and statins can accumulate and result in drug toxicity. Caution should be taken before administering these drugs (García et al., 2021).This patient was given statin therapy for lipid lowering therapy so that the side effects of statins should be monitored properly

Several mechanisms have been proposed highlighting the protective effects of bilirubin: (1) Bilirubin has antioxidant properties independent of whether it is free or albumin bound, conjugated or unconjugated. Bilirubin increases in response to the oxidative stress and acts as a scavenger of the reactive oxygen species ((Liao, 2015); (Wu et al., 1996)). Bilirubin sub-fractions (Bu and Bc) have demonstrated inhibition of low-density lipoproteins oxidation, which in turn retards the peroxidation of lipids, hence could potentially restrict the progression of atherosclerosis (T.-W. Wu et al., 1996). (2) Bilirubin has been shown to be inversely associated with increased arterial stiffness (Tamadu et al., 2015; (Liu et al., 2015)). This effect to be mediated by preservation of vascular nitric oxide, which mediates endothelial relaxation inhibits leukocyte adhesion to endothelium, vascular smooth muscle cell migration and proliferation, platelet aggregation and neointimal formation ((Pae et al., 2010); (Liu et al., 2015); (Kang et al., 2014)). (3) Bilirubin has also been seen to reduce platelet aggregation. (4) Bilirubin and heme oxygenase exhibit anti-inflammatory properties and prevent oxidant induced microvascular leukocyte adhesion. Heme oxygenase (the rate-limiting enzyme in bilirubin production) also functions as a vasodilatory and antiproliferative agent during vascular injury (Kang et al., 2014). Bilirubin has also been seen to exhibit anticomplement effect in vitro, thus, conferring protection against increased thrombogenicity and clot formation ((Kundur et al., 2015); (Rapoport et al., 2002); (Akboga et al., 2015)). Preclinical studies on mice have demonstrated a protective effect of bilirubin on angiotensin-II induced hypertension. (6) Bilirubin has 
also been described to solubilize cholesterol and promotes its clearance through the bile ((Routh, 1976); (Grendell \& Ockner, 1982)).

Several studies have suggested a cardioprotective role of bilirubin. Studies by Schwertner et al. and Song et al. provided convincing evidence of low bilirubin to be an independent risk factor for CAD (Schwertner et al., 1994). Results reported by Akboga et al., Wei et al., and Stojanov et al., further helped to establish an inverse association of bilirubin levels with the risk of developing CAD. Study by Erdogan et al. in 2012, showed that higher serum bilirubin levels were associated with good collateral development as compared to poor collateral development. These findings suggest a possible protective effect of elevated serum bilirubin levels against myocardial ischemia in patients with chronic total coronary occlusion with collaterals limiting infarct size and providing additional blood flow to the ischemic area (Erdogan et al., 2012). Wei et al. also showed similar results with a significant inverse correlation between CAD and total bilirubin in patients who underwent coronary angiography (Wei et al., 2012).

Contrary to the evidence presented above, several studies have negated the protective effect of bilirubin on CAD. British Regional Health Study (BRHS) was a prospective study designed to examine the relationship between the level of bilirubin and risk of ischemic CAD. A U-shaped relationship was observed between serum bilirubin and risk of ischemic CAD with increased risk at bilirubin concentrations $<0.4$ $\mathrm{mg} / \mathrm{dL}$ and at $>0.7 \mathrm{mg} / \mathrm{dL}$. Ayaz et al. reported an independent positive association between bilirubin and left ventricular mass hypertrophy in a population with untreated hypertension. After performing a linear and logistical regression, total bilirubin was shown to be an independent risk factor for CAD (Ayaz et al., 2014). The authors attribute this to the suppression of reactive oxygen species (Ndisang \& Jadhav, 2009). In a population of ST-elevation myocardial infarction, demonstrated an increased mortality rate and major adverse cardiac events in patients with elevated bilirubin level (Acet et al., 2014). Whether bilirubin is a by-product of another reaction with cardioprotective effects, or whether it has a direct role in reducing severity of CAD remains to be elucidated (Gupta et al., 2017).

Studies have shown that the protective effects of bilirubin are additive or synergistic to highdensity lipoprotein (HDL)-cholesterol and when HDL-cholesterol and bilirubin levels are combined in ratios of low-density lipoprotein (LDL)-C/(HDL-C+bilirubin) and cholesterol/(HDL-C+bilirubin), a 3\% increase in predictive value of CAD is observed (Wagner et al., 2015); (Schwertner \& Fischer Jr, 2000). Thus, given the additive nature of bilirubin to other risk factors, its cardioprotective effects might get masked in the presence of other unfavorable risk factors such as hypertension, diabetes, and hyperlipidemia, as seen the study population. These findings are further supported by a comparatively lower prevalence of these risk factors (hypertension, diabetes, and hyperlipidemia) in studies supporting a positive correlation among reduced bilirubin levels with increased risk of CAD ((Hopkins et al., 1996); (Mahabadi et al., 2014); (Song et al., 2014)).

In the largest study in the United States, which aims to determine effects of elevated bilirubin on $\mathrm{CAD}$ and also aims to determine predictors of CAD in patients with abnormal bilirubin excretion. There was increased prevalence of CAD in patient population with increased prevalence of cardiovascular risk factors. Age, diabetes mellitus, hypertension, and hyperlipidemia were found to be independent predictors 
of CAD. The increased prevalence of cardiovascular risk factor might mask the protective effects of bilirubin, finding that needs further investigation (Gupta et al., 2017).

While the available evidence regarding the effects of bilirubin are not definitive and different studies provide contradictory findings, some useful conclusions can be drawn. First, it is possible that the protective effects seen with higher bilirubin levels are possibly mediated through heme oxygenase or by other substrates involved in the pathway of bilirubin production, namely, biliverdin and carbon monoxide. Although few studies have reported an inverse association between bilirubin and the risk of CAD, no such association was seen with UGT1A1 gene polymorphism and the risk of CAD. Thus, a conclusion can be safely inferred that if at all bilirubin is protective in CAD, it is likely that bilirubin production (by induction of heme oxygenase and accompanied by production of carbon monoxide) and not just its excretion indirectly confers the protective effect observed with CAD. This would reflect as bilirubin having a protective effect on CAD whereas, in reality it is only a mediator or a marker (Gupta et al., 2017). Second, low bilirubin levels can be indicative of decreased heme oxygenase activity (a powerful antioxidant) or could be indicative of high oxidative stress in patients leading to consumption of the natural anti-oxidants including bilirubin. Hence, lower levels of bilirubin are perhaps not the causal factor for CAD but may indicate patients at an increased risk of developing CAD (Wu et al., 2009). Third, bilirubin requires vitamin $\mathrm{E}$ as the co-oxidant, hence patients with a high bilirubin and deficiency of vitamin $\mathrm{E}$, have less atheroprotective effect that weakens the inverse association between elevated bilirubin levels and the risk of CAD (Neuzil \& Stocker, 1994). Also, Grosser et al have reported induction of heme oxygenase with statin and aspirin therapy. Induction of heme oxygenase increases the bilirubin production. Individuals with UGT1A1 gene variants have a lower capability of exclusion of bilirubin; hence, bilirubin accumulation is to be expected in individuals on statins and aspirin. Hence as per this hypothesis, patients with CAD or at an increased risk of CAD with UGT1A1 gene polymorphism, on aspirin and statin should have increased levels of bilirubin. In that case, bilirubin might be looked upon as a marker of the drug ((Grosser et al., 2003); (Grosser et al., 2004)).

Mendelian randomization is done to establish a causal relationship (Relton \& Davey Smith, 2012). As mentioned above, a lack of significant association between the gene polymorphisms of UGT1A1 and risk for $\mathrm{CAD}$ goes in favor of bilirubin being a marker than a primary mediator for the cardioprotective effects observed with CAD. Moreover, it also points out towards incomplete penetrance of the UGT1A1 gene. Inconsistent results further support the need for further exploration of the underlying mechanisms and a prospective study with a high power to establish a definite causal relationship between bilirubin levels and CAD (Kireyev et al., 2016).

\section{Conclusion}

This patient suffered from coronary heart disease despite experiencing chronic hyperbilirubinemia due to Rotor syndrome. Patients have risk factors for CAD, namely hypertension and dyslipidemia. The protective effects of bilirubin are not proven in this patient.

\section{Acknowledgements}


The authors would like to thank to the patient and his caregivers who allowed this case to be documented.

\section{References}

Acet, H., Ertş, F., Akıl, M. A., Polat, N., Aydın, M., Akyüz, A., Ayçiçek, H., \& Alan, S. (2014). A novel predictor of infarct-related artery patency before percutaneous intervention and in-hospital outcomes for ST-segment elevation myocardial infarction patients: serum bilirubin level. Postepy w Kardiologii Interwencyjnej= Advances in Interventional Cardiology, 10(2), 91.

Akboga, M. K., Canpolat, U., Sahinarslan, A., Alsancak, Y., Nurkoc, S., Aras, D., Aydogdu, S., \& Abaci, A. (2015). Association of serum total bilirubin level with severity of coronary atherosclerosis is linked to systemic inflammation. Atherosclerosis, 240(1), 110-114.

Ayaz, T., Durakoğlugil, M. E., Kocaman, S. A., Durakoğlugil, T., Erdoğan, T., Şahin, O. Z., Şahin, S. B., Çiçek, Y., \& Şatiroğlu, Ö. (2014). Bilirubin level is associated with left ventricular hypertrophy independent of blood pressure in previously untreated hypertensive patients. Korean Circulation Journal, 44(5), 336-343.

Dhumeaux, D., \& Erlinger, S. (2013). Hereditary conjugated hyperbilirubinaemia: 37 years later. Journal of Hepatology, 58(2), 388-390.

Erdogan, B., Bauer, T. N., Truxillo, D. M., \& Mansfield, L. R. (2012). Whistle while you work: A review of the life satisfaction literature. Journal of Management, 38(4), 1038-1083.

Erlinger, S., Arias, I. M., \& Dhumeaux, D. (2014). Inherited disorders of bilirubin transport and conjugation: new insights into molecular mechanisms and consequences. Gastroenterology, 146(7), 1625-1638.

García, A. C., García, D. R.-C., Ramirez, A. L., Palacios, B. A., Alba, C. G., David, R.-C. G., \& Alba, L. R. (2021). Dubin-Johnson syndrome as a laparoscopic finding. Revista Espanola de Enfermedades Digestivas: Organo Oficial de La Sociedad Espanola de Patologia Digestiva, 113(8), 610-611.

Grendell, J. H., \& Ockner, R. K. (1982). Mesenteric venous thrombosis. Gastroenterology, 82(2), 358-372.

Grosser, N., Abate, A., Oberle, S., Vreman, H. J., Dennery, P. A., Becker, J. C., Pohle, T., Seidman, D. S., \& Schröder, H. (2003). Heme oxygenase-1 induction may explain the antioxidant profile of aspirin. Biochemical and Biophysical Research Communications, 308(4), 956-960.

Grosser, N., Hemmerle, A., Berndt, G., Erdmann, K., Hinkelmann, U., Schürger, S., Wijayanti, N., Immenschuh, S., \& Schröder, H. (2004). The antioxidant defense protein heme oxygenase 1 is a novel target for statins in endothelial cells. Free Radical Biology and Medicine, 37(12), 20642071.

Gupta, N., Chaudhary, R., Krishnamoorthy, P., Mahajan, S., Bodin, R., \& Sule, S. (2017). Coronary Artery Disease in Patients With Disorders of Bilirubin Excretion. American Journal of Therapeutics, 24(6), e653-e658.

Hopkins, P. N., Wu, L. L., Hunt, S. C., James, B. C., Vincent, G. M., \& Williams, R. R. (1996). Higher serum bilirubin is associated with decreased risk for early familial coronary artery disease. Arteriosclerosis, Thrombosis, and Vascular Biology, 16(2), 250-255.

Kagawa, T., Adachi, Y., Hashimoto, N., Mitsui, H., Ohashi, T., Yoneda, M., Hasegawa, I., Hirose, S., Tsuruya, K., \& Anzai, K. (2017). Loss of organic anion transporting polypeptide 1B3 function causes marked delay in indocyanine green clearance without any clinical symptoms. Hepatology (Baltimore, Md.), 65(3), 1065.

Kang, S. J., Lee, C., \& Kruzliak, P. (2014). Effects of serum bilirubin on atherosclerotic processes. Annals of Medicine, 46(3), 138-147.

Kireyev, P., Pauwels, K., \& Gupta, S. (2016). Do display ads influence search? Attribution and dynamics in online advertising. International Journal of Research in Marketing, 33(3), 475-490. https://doi.org/10.1016/j.ijresmar.2015.09.007

Kundur, A. R., Singh, I., \& Bulmer, A. C. (2015). Bilirubin, platelet activation and heart disease: a missing link to cardiovascular protection in Gilbert's syndrome? Atherosclerosis, 239(1), 73-84.

Liao, S.-L. (2015). The role of bilirubin and phototherapy in the oxidative/antioxidant balance. Pediatrics \& Neonatology, 56(2), 77-78.

Liu, J., Wang, L., Tian, X. Y., Liu, L., Wong, W. T., Zhang, Y., Han, Q.-B., Ho, H.-M., Wang, N., \& Wong, S. L. (2015). Unconjugated bilirubin mediates heme oxygenase-1-induced vascular benefits in diabetic mice. Diabetes, 64(5), 1564-1575.

Mahabadi, A. A., Lehmann, N., Möhlenkamp, S., Kälsch, H., Bauer, M., Schulz, R., Moebus, S., Jöckel, 
K.-H., Erbel, R., \& Heusch, G. (2014). Association of bilirubin with coronary artery calcification and cardiovascular events in the general population without known liver disease: the Heinz Nixdorf Recall study. Clinical Research in Cardiology, 103(8), 647-653.

Memon, N., Weinberger, B. I., Hegyi, T., \& Aleksunes, L. M. (2016). Inherited disorders of bilirubin clearance. Pediatric Research, 79(3), 378-386.

Ndisang, J. F., \& Jadhav, A. (2009). Upregulating the heme oxygenase system suppresses left ventricular hypertrophy in adult spontaneously hypertensive rats for 3 months. Journal of Cardiac Failure, 15(7), 616-628.

Neuzil, J., \& Stocker, R. (1994). Free and albumin-bound bilirubin are efficient co-antioxidants for alphatocopherol, inhibiting plasma and low density lipoprotein lipid peroxidation. Journal of Biological Chemistry, 269(24), 16712-16719.

Pae, H.-O., Son, Y., Kim, N.-H., Jeong, H. J., Chang, K. C., \& Chung, H.-T. (2010). Role of heme oxygenase in preserving vascular bioactive NO. Nitric Oxide, 23(4), 251-257.

Pratt, E., Sissung, T. M., \& Figg, W. D. (2012). Loss of OATP1B3 function causes Rotor syndrome: implications for potential use of inhibitors in cancer. Cancer Biology \& Therapy, 13(14), 13741375.

Rapoport, M., Dawson, H. N., Binder, L. I., Vitek, M. P., \& Ferreira, A. (2002). Tau is essential to $\beta$ amyloid-induced neurotoxicity. Proceedings of the National Academy of Sciences, 99(9), 63646369.

Relton, C. L., \& Davey Smith, G. (2012). Two-step epigenetic Mendelian randomization: a strategy for establishing the causal role of epigenetic processes in pathways to disease. International Journal of Epidemiology, 41(1), 161-176.

Routh, J. L. function. (1976). 1n: Tietz NW (eds.). Fundamentals of clinical chemistry. Philadelphia: WB Saunders, 1026-1062.

Schwertner, H. A., \& Fischer Jr, J. R. (2000). Comparison of various lipid, lipoprotein, and bilirubin combinations as risk factors for predicting coronary artery disease. Atherosclerosis, 150(2), 381387.

Schwertner, H. A., Jackson, W. G., \& Tolan, G. (1994). Association of low serum concentration of bilirubin with increased risk of coronary artery disease. Clinical Chemistry, 40(1), 18-23.

Shimizu, Y., Naruto, H., Ida, S., \& Kohakura, M. (1981). Urinary coproporphyrin isomers in Rotor's syndrome: a study in eight families. Hepatology, 1(2), 173-178.

Song, Y. S., Koo, B. K., Cho, N. H., \& Moon, M. K. (2014). Effect of low serum total bilirubin levels ( $\leq$ $0.32 \mathrm{mg} / \mathrm{dl}$ ) on risk of coronary artery disease in patients with metabolic syndrome. The American Journal of Cardiology, 114(11), 1695-1700.

Stojanov, M., Stefanovic, A., Dzingalasevic, G., Ivanisevic, J., Miljkovic, M., Mandic-Radic, S., \& Prostran, M. (2013). Total bilirubin in young men and women: association with risk markers for cardiovascular diseases. Clinical Biochemistry, 46(15), 1516-1519.

van de Steeg, E., Stránecký, V., Hartmannová, H., Nosková, L., Hřebíček, M., Wagenaar, E., van Esch, A., de Waart, D. R., Elferink, R. P. J. O., \& Kenworthy, K. E. (2012). Complete OATP1B1 and OATP1B3 deficiency causes human Rotor syndrome by interrupting conjugated bilirubin reuptake into the liver. The Journal of Clinical Investigation, 122(2), 519-528.

Wagner, K.-H., Wallner, M., Mölzer, C., Gazzin, S., Bulmer, A. C., Tiribelli, C., \& Vitek, L. (2015). Looking to the horizon: the role of bilirubin in the development and prevention of age-related chronic diseases. Clinical Science, 129(1), 1-25.

Wei, S., Gao, C., Wei, G., Chen, Y., Zhong, L., \& Li, X. (2012). The level of serum bilirubin associated with coronary lesion types in patients with coronary artery disease. Journal of Cardiovascular Medicine, 13(7), 432-438.

Wu, T.-C., Lin, C.-H., \& Shiau, S.-Y. (2009). Developing measures for assessing the causality of safety culture in a petrochemical industry. Water, Air, \& Soil Pollution: Focus, 9(5-6), 507.

Wu, T.-W., Fung, K. P., Wu, J., Yang, C.-C., \& Weisel, R. D. (1996). Antioxidation of human low density lipoprotein by unconjugated and conjugated bilirubins. Biochemical Pharmacology, 51(6), 859862. 